Itinéraires Itinéraires

Littérature, textes, cultures

Numéro inaugural | 2008

L'homme en tous genres

\title{
« Ils se marièrent... etc. » : Chouchou, La Confusion des genres et autres mariages
}

\section{Mireille Rosello}

\section{OpenEdition}

\section{Journals}

Édition électronique

URL : http://journals.openedition.org/itineraires/2225

DOI : $10.4000 /$ itineraires.2225

ISSN : 2427-920X

Éditeur

Pléiade

\section{Édition imprimée}

Date de publication : 1 décembre 2008

Pagination : 191-204

ISBN : 978-2-296-07519-1

ISSN : 2100-1340

\section{Référence électronique}

Mireille Rosello, « «Ils se marièrent... etc. » : Chouchou, La Confusion des genres et autres mariages », Itinéraires [En ligne], Numéro inaugural | 2008, mis en ligne le 01 décembre 2008, consulté le 05 mai 2019. URL : http://journals.openedition.org/itineraires/2225; DOI : 10.4000/itineraires.2225

\section{(C) $(\oplus \Theta$}

Itinéraires est mis à disposition selon les termes de la licence Creative Commons Attribution - Pas d'Utilisation Commerciale - Pas de Modification 4.0 International. 


\title{
" Ils se marièrent... etc. » : Chouchou, La Confusion des genres et autres mariages
}

\begin{abstract}
In the context of international debates on gay marriages, this article looks at three visual narratives that invite us to reflect on the issues of masculinity and norms. It focuses on the unique and specific ways in which a wedding between two men in a comedy (Chouchou) or in a public hoax (Coluche and Thierry Le Luron) as well as a queer wedding between a man and a woman (La Confusion des genres) reinforce or critique heterosexual social and gender norms.
\end{abstract}

Cet article se propose de regarder de près trois mariages, ou plutôt trois représentations d'une cérémonie que le spectateur est censé reconnaître comme un mariage tout en les repérant comme des mariages bizarres, décalés ou dissidents, qui testent les limites de ce que J. L. Austin nous a appris à repérer comme les règles qui transforment un rituel en acte performatif ${ }^{1}$. Une de ces règles fait de la rencontre entre un homme et une femme une évidence qui n'a même pas besoin de se dire ${ }^{2}$. Or, les trois exemples sont des

1. Au début de son How to Do Things with Words, le philosophe choisit trois exemples de ce qu'il entend par ces actes de parole performatifs qui ne relèvent pas d'une logique du « vrai ou faux » mais de la précision avec laquelle les règles de la prise de parole (ce que l'on pourrait appeler une loi du genre) sont respectées. Alors l'élocution coïncide avec l'événement que l'on fait advenir. Le tout premier de ces exemples est justement celui de la cérémonie du mariage : "I do (sc. take this woman to be my lawful wedded wife)" - as uttered in the course of the marriage ceremony ", J. L. Austin, How to Do Things with Words, $2^{\mathrm{e}}$ éd., Oxford et New York, Oxford University Press, 1975, p. 5.

2. Ainsi, lorsqu'Austin nous propose des exemples de ce que pourrait être un acte performatif qui échoue, il choisit comme critère non pas la différence de sexe entre les époux mais le partage de la religion: si l'un est musulman et l'autre chrétien, la communauté ne reconnaîtra pas les mêmes scripts comme "réussis ", Austin, op. cit., p. 27. L'intérêt du non-dit de l'obligation hétérosexuelle est évidemment que l'on peut s'appuyer sur cette absence de dit (aucun des articles du Code civil n'est explicite à ce sujet) pour récuser la 
récits visuels complexes qui mettent en scène la masculinité des marié(e)s et nous invitent à lire et regarder la façon dont cette représentation s'effectue. Ces images et les constructions narratives de cette mise en question de la masculinité s'ajoutent à notre imaginaire, c'est-à-dire au réservoir de mythes dans lequel nous puisons, mais peut-être sans savoir comment, lorsque le contexte fait naître le besoin d'une interprétation, d'une analyse ou même d'une décision politique et éthique de ce qui touche au masculin, et notamment dans le cas des cérémonies qui supposent la présence d'une masculinité générale, abstraite et non préalablement définie.

Commençons l'analyse des trois objets narratifs en remarquant que les deux premiers mariages unissent deux hommes (mais que ce ne sont pas des mariages gay) et que le troisième est célébré entre un homme et une femme (mais qu'il est probablement le plus queer des trois), car l'intérêt et des trois récits et des discours qui les enserrent dans un contexte scientifique est justement de souligner les premières formulations comme hâtives. Il serait donc plus prudent de décrire ces trois mariages décalés comme une sorte de laboratoire imaginaire qui nous permet de tester le rapport instable entre un individu et le genre masculin ou féminin, mais aussi le rapport entre les lois narratives et les normes du genre (au sens d'identité sexuée) et la façon dont nous nous sentons libres ou prisonniers par rapport à une règle. En général, ces normes se renforcent mutuellement pour fabriquer un principe de vraisemblance auquel nous croyons avoir toujours déjà adhéré et qui nous dit qu'un être humain est, une bonne fois pour toutes et dans tous les contextes, masculin ou féminin, que le mariage (en France en tout cas) implique toujours la rencontre des deux, et que la représentation d'un mariage fictif doit ressembler, pour être crédible, à ce que serait la performance réussie d'un mariage. Les trois mariages suggèrent qu'il est quasiment impossible de résister à un seul de ces théorèmes à la fois et que chaque décalage d'une des normes modifie la façon dont la masculinité est gérée par les autres.

Le premier mariage a lieu à la fin de Chouchou, le film de Merzak Allouache qui a, en 2003, attiré plus de trois millions de spectateurs. Les mariés sont incarnés par Gad Elmaleh et Alain Chabat. Le premier joue le rôle de Choukri dit « Chouchou », immigré algérien qui se fait (mal) passer pour un réfugié chilien avant d'abandonner son ridicule déguisement et les quelques mots d'espagnol qu'il connaît. Il est beaucoup plus convaincant habillé en femme quand il travaille chez une psychanalyste pendant la journée et dans un cabaret pendant la nuit. C'est à L'Apocalypse, où il chante, danse et sert à boire dans ses resplendissants costumes féminins, 
que Chouchou rencontre Stanislas, dont la masculinité conventionnelle est quasi invisible en tant qu'élément de caractérisation. Ses vêtements classiques et l'absence de tout décalage par rapport à ce que l'on imagine être une attitude masculine traditionnelle sont d'ailleurs beaucoup moins importants, pour le définir au sein du récit, que son côté vieille France et son milieu socioéconomique, qui le place à mille lieues de celui de son futur époux (un dîner de famille fait de Chouchou l'étranger ignorant dont les bourdes comiques ne font que le rendre plus attachant, y compris pour sa future belle-famille).

Les dernières scènes du film ont lieu juste avant le mariage des deux hommes. La caméra réunit un public que les frontières sociales nous font en général traiter de disparate, et qui rassemble en une seule communauté la famille de Stanislas, les amis de Chouchou et les spectateurs. Tous attendent avec inquiétude (le public y compris), craignant que l'absence de la mariée à ce moment fatidique ne soit causée par la déportation dont le personnage était menacé. Le film joue du topos classique du mariage quasi célébré mais qui menace pourtant de capoter au dernier moment pour une raison ou pour une autre, et choisit finalement le genre du conte de fée en faisant apparaître une spectaculaire robe de mariée, une perruque rouge, un maquillage voyant, habités par un(e) Chouchou rayonnant(e) qui court, au ralenti, vers l'assemblée prête à l'accueillir à bras ouverts.

La deuxième cérémonie qui unit fictivement deux hommes n'appartient pas au monde du cinéma mais à celui du canular médiatisé. En 1985, Thierry Le Luron et Coluche s'unissaient, en public, «pour le meilleur et pour le rire » mais surtout pour se moquer du journaliste Yves Mourousi qui venait de se marier ${ }^{3}$. La parodie affirmait sans ambiguïté que les deux professionnels du rire jugeaient que le mariage du journaliste avait été dénaturé. Officiellement, ils critiquaient un excès de publicité qui rendait le mariage aussi «bidon» que le leur. Les caméras, invitées à se poster sur leur route, enregistraient l'arrivée des deux mariés qui avaient chacun adopté le costume le plus conventionnel qui soit par rapport à la norme hétérosexuelle. Thierry Le Luron était en costume et chapeau gris; Coluche tirait des mètres de tulle blanc et arborait une perruque blonde et une jarretière récalcitrante qu'il était obligé de remonter complaisamment au-dessus de son genou sous l'œil des caméras. Le nouveau marié déclarait entre autres: « Notre mariage est bidon nous n'avons pas peur de le dire, nous, et nous n'espérons pas avoir beaucoup d'enfants mais beaucoup d'articles et de photos dans les journaux. »

L'intérêt de ce genre de parodie est de soulever le problème de ce que l'on pourrait appeler des parodies gigognes. Le mariage des deux hommes est bien sûr «bidon» (et cette évidence est complaisamment soulignée)

3. Différents extraits filmés sont publiés sur le site <http://www.thierryleluron.net> (consulté en décembre 2007). 
parce que la cérémonie unit deux hommes. Ici, le travestissement ne sert pas à faire passer un homme pour une femme et n'est pas fait pour fabriquer du secret. Au contraire, le déguisement de Coluche est raté, grossier, revendiqué comme tel. Il pourrait servir à confirmer toutes les opérations normatives qui reposent sur des mythes de masculinité biologique (le travestissement rappelle ici les principes d'assimilation toujours ratée: le sujet dominé ou colonisé dont on exige qu'il s'assimile ne fait jamais que confirmer son absence d'authenticité). En d'autres termes, que le ratage de la performance soit assumé ou subi, le travestissement, dans ce cas, ne servirait qu'à renforcer la norme hétérosexuelle du mariage en affirmant, avec la plus solide mauvaise foi, que tout mariage entre hommes est grotesque parce que celui-là l'est. Pourtant la déclaration du marié compare cette parodie à un autre vrai mariage, un mariage tout à fait légal puisque les participants n'ont transgressé aucune des règles qui régissent la performance et transforment la parole en acte. Le « nous n'hésitons pas à le dire, nous » oppose donc, non pas un mariage «bidon » à un mariage respectable, convenable, mais une parodie honnête (qui se dit parodie) à une parodie hypocrite (qui se prétend officielle et légale). Le côté farce de la cérémonie entre hommes souligne qu'ils sont soustraits (en tant qu'acteurs) à l'ordre du performatif mais aussi que le mariage de Mourousi fait lui aussi partie de ces discours théâtraux qui annulent la valeur de la procédure ( $c f$. Austin, op. cit., p. 8-9). Tous sont des professionnels qui ont visiblement monté un coup médiatique: "Qu'on ne nous accuse pas surtout de nous moquer d'une institution, d'autres que nous vont s'en charger bientôt plus sérieusement. » La façon dont la norme est parodiée met donc en lumière que certaines formes de transgression sont prises, et peut-être à tort, plus au sérieux que d'autres, une suggestion que l'on peut garder à l'esprit chaque fois que le mot de «parodie » est utilisé pour condamner systématiquement les mariages qui ne correspondent pas aux règles de l'hétérosexualité. À Patrick Poivre d'Arvor, qui lui demandait, au cours d'un entretien, quelle était sa position sur «le mariage homosexuel», Nicolas Sarkozy répondait, en 2004 :

Je crois qu'il ne faut pas mélanger les genres et, il y a des problèmes, chacun le sait, qui doivent être résolus. On a créé le PACS. C'est parfait. À l'expérience, il apparaît que ce PACS pourrait être amélioré et je souhaite qu'on l'améliore, de façon à ce que les droits et devoirs des personnes de même sexe qui vivent ensemble ou qui ont fait le choix de vivre ensemble soient respectés, au même titre que ceux des autres. Cela ne doit pas nous conduire à une parodie de mariage ${ }^{4}$.

4. P. Poivre d'Arvor et A. Chabot, «Entretien avec Nicolas Sarkozy, Président de la République française (14 juillet 2004) », <http://www.u-m-p-paris.org/article.php3 ?id_article $=292>$ (consulté en décembre 2007). 
Le paradoxe de ce canular, monté plus de dix ans avant la création du pacs, vient donc du fait que l'absence de tout lien amoureux ou sexuel entre les partenaires et la volonté délibérée de souligner la parodie réussissent en fait à respecter l'institution du mariage dans son ensemble. La parodie souligne que les transgressions les plus visibles (notamment la présence de deux masculinités plaquées sur des représentations masculines et féminines) ne sont pas nécessairement celles qui constituent la plus sérieuse mise en cause du rituel.

$\mathrm{Au}$ contraire, La Confusion des genres d'Ilan Duran Cohen (2000) interroge directement le non-dit du mariage alors que le type de cérémonie qui y est décrite pourrait, justement, être protégée par les normes dont Thierry Le Luron et Coluche montraient qu'elles ne sont pas mises en danger par la parodie. Par rapport aux deux premiers mariages où le spectaculaire et le comique se rejoignent et posent donc la question des dissidences par démesure, excès ou ironie, celui qui se déroule dans La Confusion des genres serait, à première vue, du côté de la sobriété. Des trois mariages, c'est le seul à ne pas mettre en scène deux hommes au moment de la cérémonie. C'est le seul que l'on pourrait prendre au sérieux d'un point de vue intradiégétique (il se déroule à la mairie, les conditions sont réunies pour que l'acte de parole soit fictivement légal). Mais il est aussi le seul où le rapport entre le script de la cérémonie et les présupposés culturels qu'il est censé confirmer est directement interrogé. Comparés au bon déroulement de la cérémonie, et la norme hétérosexuelle et le cliché de l'histoire d'amour comptent peu. Le héros du film, Alain (Pascal Greggory), s'apprête à épouser Laurence, son amie et patronne (Nathalie Richard). Il ne l'aime pas, il ne la désire pas et les deux personnages sont explicites sur ce point. À aucun moment, le scénario n'a besoin de l'idée de secret. Aucun des héros n'essaie de se faire passer pour autre qu'il n'est (autre sexe ou autre préférence sexuelle). Simplement, pour Alain, cette apparente sincérité ne résout pas le problème puisqu'il est incapable de nommer, de cerner ou de comprendre ses propres désirs (multiples). Les aligner sur un sexe en particulier n'a aucun sens mais il n'est pas non plus enclin à se représenter comme gay. Il sera le père de l'enfant de Nathalie, tout en étant l'amant du jeune Christophe, qui habite chez lui, et où les rejoindront provisoirement Babette (Julie Gayet), une jeune coiffeuse que le héros affirme aimer, elle aussi, « à sa manière », faute de pouvoir nommer autrement la « confusion » qu'il subit, impose et généralise.

La scène du mariage elle-même donne l'occasion à tous les témoins de la cérémonie de formuler tout haut ce que l'on passe en général sous silence: la norme exige que le script vienne au secours de l'absence de ce que la mariée appelle " les belles histoires » : ils sont là pour sceller un pacte devant la représentante de l'État qui a revêtu son écharpe bleublanc-rouge, et devant les membres de familles déjà rendues légitimes par d'autres mariages. Le rôle d'Alain dans cette scène est de modifier le script 
et de dire, tout haut, ce qu'il devrait taire hypocritement. Quittant le bras de sa future épouse, il déclare à sa mère, assise derrière lui: « Maman, je suis amoureux du frère de Marlène » (elle aussi présente à la mairie). La réaction de sa mère est instantanée: «Ce n'est pas une raison pour faire attendre tout le monde. » Ce qui pourrait être amusant et libérateur ici (l'indifférence du parent face à un coming out qui devrait avoir un fort coefficient perturbateur) est en fait présenté comme la tyrannie d'une autre règle. Faire attendre est une atteinte indécente au protocole. En d'autres termes, ce qui est choquant, anormal, ce n'est pas que la cérémonie inaugure une hypocrisie mais qu'elle se déroule mal, qu'il y ait un contretemps, une erreur de parcours. Cette réponse comique mais présentée comme absolument sérieuse préfigure alors tout ce que les autres personnages vont dire l'un après l'autre pour remettre la cérémonie sur les rails, pour s'assurer que ce mariage, auquel ils ont déjà donné leur accord (visiblement en toute connaissance de cause), ait bien lieu. «Ferme les yeux et pense à lui », recommande Marlène, la sœur de l'amant d'Alain. Même son de cloche de la part de la mère et du père d'Alain qui ne semblent pas sensibles à l'ironie de leur écho: " Dans ces cas-là je ferme les yeux et je plonge », assure le père. Et son épouse de confirmer: «C'est ce qu'on a fait avec ton père. " Contrairement à Chouchou qui célèbre la possibilité d'un mariage entre hommes dont l'un souhaite occuper le rôle d'une certaine féminité mais contrairement aussi au mariage de Coluche et de Thierry Le Luron dont l'union burlesque se veut une défense de l'institution telle qu'elle est implicitement reconnue, le film d'Ilan Duran Cohen souligne la performativité arbitraire de tout mariage. Le script est souverain mais il ne vaut que parce qu'il est méticuleusement suivi, quel que soit le rapport qu'entretiennent les sujets présents avec les personnages que le scénario leur impose (homme, femme, féminisé ou non dans leur apparence ou leur identité, homosexuel-le, hétérosexuel-le ou « confus-e », amoureux/euse ou non).

Les trois objets ne se présentent pas comme une critique ni même un commentaire sur les règles qui font qu'un mariage est légal ou irrecevable mais ils sont suffisamment décalés par rapport à la norme pour provoquer la réflexion. La comparaison entre les décalages différents que ces trois mariages se permettent par rapport à la norme met en lumière les différents paramètres que l'on met en jeu, très souvent sans même les formuler, lorsqu'on invoque, décrit ou reconnaît la masculinité en général. Les trois récits nous rappellent que l'on ne pourrait pas entretenir l'illusion d'une définition même plurielle de la masculinité, si nous n'étions pas habitués à ranger arbitrairement sous sa bannière abstraite, des moments, des pratiques ou des phénomènes qui ne sont qu'épisodiquement pertinents, et toujours instables, à savoir le travestissement, l'homosexualité et la transsexualité ou transidentité.

C'est en décrivant la manière dont ces éléments s'articulent que l'on peut distinguer entre les exemples mais aussi formuler la distance qui se 
creuse entre chaque récit et les présuppositions stéréotypées que la norme nous invite à substituer au cas particulier. La comparaison entre trois couples fait naître des moments de masculinisation, c'est-à-dire non pas des catégories nouvelles mais des mises en contact et en relation différentes entre certains paradigmes.

Chouchou et La Confusion des genres ont donc en commun la représentation fictive d'une masculinité homosexuelle mais le deuxième héros ne lie pas cette masculinité-là à son mariage. Dans le canular, au contraire, la masculinité des deux hommes est dissociée de leur sexualité (mise en scène). Coluche fonctionne comme l'amante et la mariée. Mais sa mise en scène n'a pas besoin d'être ressemblante. Coluche est dans la position de femme parce que la cérémonie réclame un actant de sexe féminin mais il y a théâtralisation d'une féminisation et non masculinisation de la position féminine dans la cérémonie. Sa sexualité est non pertinente, elle est normativement supposée (à tort ou à raison) comme hétérosexuelle, ce qui implique que l'autre membre du couple est supposé, là aussi à tort ou à raison, jouer le rôle du masculin hétérosexuel dont la performance est minimisée (c'est-à-dire que sa masculinité semble "neutre » au regard de la féminisation grotesque de Coluche). Si ce mariage était un mariage " gay ", l'image et le récit ne nous le montrerait pas ${ }^{5}$. De même, Chouchou n'est qu'en apparence un mariage gay au sens restreint du terme (deux partenaires sexuels du même sexe biologique sont unis), c'est plutôt un mariage de conte de fées où la possibilité pour Chouchou de se métamorphoser en femme comme Cendrillon se transforme en princesse n'est ni plus ni moins invraisemblable que la possibilité pour deux hommes de se marier en grande pompe, en robe blanche et perruque rouge. Certes, les deux partenaires sont deux hommes et ils sont amants mais le film ne s'intéresse nullement au problème de l'impossibilité historique de la performance du mariage entre deux masculinités. Ici, la masculinité travestie ne sert pas à parodier le mariage, encore moins l'image caricaturée d'homosexuels dits efféminés.

Mettre l'accent sur les contraintes qu'impose le genre revient en fait à se libérer des préjugés qui parfois limitent des récits gouvernés par des normes similaires mais que nous ne reconnaissons pas parce que nous évoluons dans un contexte identifié comme politique. Par exemple, si je choisis de regarder Chouchou dans l'optique d'une réflexion sur les mariages gay, la fin du film risque de simplement confirmer les inquiétudes de ceux des activistes qui craignent que la lutte en faveur de la légalisation du mariage

5. Si ironie il y a vis-à-vis de ce que les spectateurs savent de la biographie des acteurs, cette couche de sens ne fait pas partie de la diégèse et crée, implicitement, un public averti qui lira autrement, mais sans éliminer l'interprétation que chacun peut faire de la critique du mariage médiatisé. 
ne devienne un phénomène à la fois choquant pour certains conservateurs et profondément conservateur lui-même ${ }^{6}$.

Dans un contexte où l'internationalisation des questions gay nous oblige à surveiller d'autres articulations (celles, par exemple, entre masculinités et féminités dissidentes et immigration ou ethnicité), la définition du mariage entre deux sujets du même sexe ne peut pas être séparée d'un débat sur les exclus de 1'assimilation. En 2004, Carl Stychin écrit par exemple:

À mon avis, on devient vite conservateur lorsqu'on parle de mariages gay ou de droits dus au partenaire de même sexe. Non seulement ce genre de démarche politique risque d'être assimilationniste mais elle a aussi pour résultat de fabriquer des «queers » qui ne méritent pas ces droits, ceux qui sont dangereux, que l'on ne peut pas civiliser. Il est bien trop tentant pour le « citoyen gay » de consommer ses droits de l'homme et de se retirer ensuite des rangs politiques progressistes, alors même que ceux qui ont accordé les droits en question passent aussi des mesures qui font fi de ces mêmes droits dans le contexte de l'immigration ou de la lutte contre le terrorisme ${ }^{7}$.

Dans un article consacré à Chouchou, Darren Waldron s'interroge aussi sur la valeur de cette union qui semble

confirmer plutôt que contester les valeurs morales dominantes. Il semble que l'institution la plus sacrée et la mieux protégée de notre société hétérosexiste soit plaquée de façon un peu simpliste sur un couple homosexuel, sans générer d'angoisse particulière. De plus, dans la mesure où Chouchou a déjà fait une allusion à son désir de se faire opérer, l'idée est qu'ils formeront un jour un couple biologiquement hétérosexuel ${ }^{8}$.

Le costume et le maquillage sont un déguisement. Chouchou s'identifie à une féminité elle-même à redéfinir. La catégorie de transgenre transparaît en filigrane. Et si outrance il y a dans certaines scènes, elle ne relève pas d'un renforcement des stéréotypes de l'homosexuel efféminé qui exagère avec affectation une norme féminine elle-même imaginaire ${ }^{9}$. Ce qui

6. Voir notamment M. Warner, The Trouble with Normal: Sex, Politics, and the Ethics of Queer Life, Cambridge (Mass.), Harvard University Press, 1999.

7. C. Stychin, «Same-Sex Sexualities and the Globalization of Human Rights Discourse », McGill Law Journal, no 49, 2004, p. 952-958 (p. 967); je traduis.

8. D. Waldron, «New Clothes for Temporary Transvestites? Sexuality, Cross-dressing and Passing in the Contemporary French Film Comedy », Modern and Contemporary France, no 14, 3, 2006, p. 347-361 (p. 44-45); je traduis. Voir aussi A. Khalil, « The Myth of Masculinity in the Films of Merzak Allouache », The Journal of North African Studies, $\mathrm{n}^{\circ} 12,3,2007$, p. 329-345.

9. Le film peut en effet faire craindre un renforcement des stéréotypes les plus conventionnels comme le montre Alain Brassart dans L'Homosexualité dans le cinéma français, Paris, Nouveau Monde Éditions, 2007. Brassart critique l'amalgame entre féminisation et homosexualité qui renforce des clichés souvent homophobes (op. cit., p. 12). 
peut être lu comme une surperformance du féminin renvoie à deux autres codes génériques: la culture camp et le conte de fées. Ces deux genres produisent des normes cohérentes auxquelles Chouchou adhère. La visite de Paris avec Stanislas, qui correspond à un moment de légitimation et de visibilité pour l'émigré algérien réduit à la clandestinité, est colorée par une vision camp qui remplace les monuments nationaux officiels par une culture décalée. Ainsi, le lieu de mémoire parisien qu'il voudra visiter en priorité est le lieu de l'accident où Lady Diana a trouvé la mort. Chouchou viendra y déposer des fleurs en femme bourgeoise (mais aussi camp) pendue amoureusement au bras de Stanislas. Le film remplace la tour Eiffel ou l'Arc de Triomphe du touriste lambda par une icône gay ${ }^{10}$. Ce n'est donc pas en exagérant une féminité abstraite voyante mais en collant à une norme claire d'un certain genre de féminité que le personnage nous amuse. Même les apparitions nocturnes à la boîte de nuit sont en fait conformistes et correspondent à cette norme-là qui réclame paillettes et maquillages spéciaux. La masculinité habillée en femme reste guidée par un script qui n'est pas subverti mais qui n'est pas grotesque même s'il est choisi en dehors du stéréotype naturalisé de la culture dominante.

La Confusion des genres décale l'opposition entre mariage gay et mariage hétérosexuel en nous montrant que cette binarité suppose une superposition trop simple entre masculinité, homosexualité et mariage: dans La Confusion des genres, le mariage n'est ni hétérosexuel ni gay. Les partenaires sexuels ne masquent pas une relation secrète par une cérémonie conventionnelle: le personnage n'épouse pas une femme pour cacher son amour pour Christophe. Cette union-là n'est pas célébrée mais elle est publique et Christophe est le premier à tenir dans ses bras l'enfant né de l'unique rapport sexuel entre l'homme et la femme. Symboliquement, il est le vrai parent à qui l'on confie le nouveau-né et qui avertit que si on le lui met dans les bras « il ne le quittera plus ». Les grands-parents biologiques sont malgré eux relégués dans la salle d'attente. Le mariage hétérosexuel n'est donc ni un mariage de convenance ni une union que les partisans au changement de la loi pourraient facilement revendiquer. Comme dans Chouchou, les deux hommes sont liés sexuellement mais leur homosexualité n'est pas l'objet sur lequel le regard de la caméra et les scripts du récit se focalisent.

Dans La Confusion des genres, aucun des deux amants n'est visiblement féminisé et le choix d'un partenaire sexuel est d'ailleurs rarement exclusif en termes de sexe biologique. Du point de vue du spectacle de leur masculinité (costume, maniérisme, stéréotypes de la féminisation), les

10. Pour une analyse de la place du camp en France, de ses racines historiques au XVII ${ }^{\mathrm{e}}$ siècle et notamment à Versailles (c'est l'art de se « camper »), et pour une analyse de la façon dont s'articule le rapport entre culture camp et féminisation en Europe et aux États-Unis, voir le troisième chapitre du livre de Jean-Yves Le Talec, Folles de France: repenser l'homosexualité masculine, Paris, La Découverte, 2008, p. 79-120. 
deux amants ont une masculinité à la visibilité conventionnelle et une homosexualité publique. Le film n'a rien de camp et ne fait jamais intervenir le travestissement d'un homme. En revanche, la problématique du rapport entre désir du même (homo) et apparence masculine ou féminine est mentionnée dans une scène qui, là aussi, nous invite à nous méfier d'un simple rapprochement entre désir pour l'homme et désir pour une apparence conventionnellement masculine. Lorsque Babette et Alain découvrent leur attirance mutuelle, le héros, comme il le fait pendant tout le film, ne sait expliquer ce qu'il ressent qu'en termes de « confusion ». Incapable de formuler ou de comprendre son propre désir, il renonce à tout schéma. Mais Babette réintroduit un script qui pourrait faire d'elle l'objet masculinisé d'un désir qu'elle suppose homosexuel et auquel elle pourrait, en se masculinisant, correspondre mieux. Une scène nous la montre soudain différente, les cheveux coupés court, son visage de « garçonne » aussi soigneusement raté en termes de performance masculine que l'était le personnage féminin de Coluche. L'effet, toutefois, n'est pas censé être comique. Simplement, la performance du masculin n'est pas réussie et contrairement au clin d'œil aux icônes gay qui apparaissent dans Chouchou, il n'y a ici aucune allusion à une imagerie butch qui ferait référence à une culture à part entière. La réaction du héros est amusante non pas parce que la masculinisation de son amante est elle-même comique mais parce qu'elle démontre l'inefficacité du récit qui amalgame apparence et désir:

«Vos cheveux, qu'est-ce que vous avez fait, vous êtes folle?

— Vous n'aimez pas?

— Mais non pas du tout!

- Ils m'ont dit que je ressemblerais à un garçon.

— On vous a dit n'importe quoi!»

Le désir de « ressembler à un garçon » dont fait preuve le personnage féminin ne s'explique ici que par le désir de plaire à un garçon qui aime les garçons. Enfermer le personnage dans cette masculinité générique qui désire une masculinité générique est une démarche elle-même potentiellement violente. C'est l'envers du miroir (ou une image déformée) de la paranoïa hétérosexuelle qui fait craindre à tout sujet d'être la proie d'homosexuels du même sexe. La tentative pour ressembler à un garçon est basée sur le même principe que la peur qui fait croire aux membres des communautés non mixtes (ceux ou celles qui partagent un même vestiaire ou un même sous-marin, un groupe traditionnellement masculin comme l'armée ou la police) qu'ils risquent sans cesse un viol physique ou mental en la présence d'homosexuels qui traiteraient tout corps du même sexe comme un objet de désir à posséder. Cette confusion entre homosexualité, sexe biologique et désir du même est également épinglée dans Chouchou de manière plus humoristique et plus traditionnelle. Dans ce film, la paranoïa classique est évoquée lorsqu'un des jeunes délinquants qui a besoin d'aide est hébergé par Chouchou et émet une vague inquiétude à l'idée que 
Chouchou l'ait fait entrer pour « faire des trucs moyenâgeux ». La violence comique avec laquelle Chouchou réagit ( Qu'est-ce que tu racontes, regarde ta gueule, tu es en travaux! ») souligne que son désir dit homosexuel ( «je suis ce que tu veux», dit-il, pour couper court aux questions) n'est pas plus générique que n'importe quel désir (d')hétérosexuel. Et qui plus est, dans le cas de son invité, toute éroticisation est d'emblée exclue par un refus de l'apparence physique du corps. L'autre est laid. Le critère est sans doute conventionnel et dans le cas des couples hétérosexuels, réduire le désir à l'apparence physique serait sans doute traité comme une attitude superficielle et particulièrement macho. Le sketch de Coluche et de Thierry Le Luron a d'ailleurs l'occasion de se moquer de cette fétichisation d'un certain type de beauté physique typiquement féminine ou féminisée. Si Le Luron fait rire quand il affirme «je préfère avoir une épouse comme Coluche qui a de la personnalité », c'est parce qu'il admet que son partenaire n'a pas réussi à jouer le rôle stéréotypé de la mariée. Au sein même du mariage «bidon » qui pourrait se permettre de reproduire les images les plus éculées sur l'opposition entre la douce et belle épouse et l'homme fort et conquérant, Le Luron nous fait rire d'un autre cliché qui donne à la femme le prix de consolation de la "personnalité » pour ne pas avoir à avouer que la beauté physique (même imitée) aurait été un atout plus présentable. Coluche est d'autant plus convaincant en parodie du mariage qu'il ne l'est pas en parodie de personnage féminin. Quant à la réaction de Chouchou, elle a l'avantage d'humilier tactiquement l'homophobe en faisant remarquer la présomption de sa crainte d'être désiré par quelqu'un qu'il exclut pouvoir lui-même un jour désirer.

Les trois scénarios, et tout particulièrement La Confusion des genres, définissent le désir comme foncièrement individualiste et lui gardent une forte marge de manœuvre par rapport aux catégories imitées ou parodiées. Le désir est imprévisible ou en tout cas il ne peut pas être déduit des schémas préexistants dans lesquels on range les pratiques ou les identités. Venant de Chouchou, inscrit dans un récit qui affirme l'identité ethnique du personnage sans l'articuler très précisément à son identité sexuée et sexuelle, la réaction est aussi une allusion intéressante à un débat plus large. Certains critiques s'inquiètent, par exemple, des conséquences de ce que l'on peut appeler la globalisation des droits de l'homosexualité (« the globalization of gay rights $»)^{11}$. Joseph Massad soupçonne les mouvements gay occidentaux et notamment anglo-saxons de faire la même erreur qu'un certain féminisme occidental qui cherchait à exporter sa bonne parole de libération et d'égalité sans se soucier de ce que cette démarche pouvait avoir d'impérialiste ou en tout cas d'eurocentré. La même critique pourrait désormais être adressée à certains discours gay globalisants qui imposent 
involontairement, dit Massad, des paramètres occidentaux et blancs à un monde qui ne reconnaît pas ces catégories, surtout lorsqu'il s'agit de « libération » par rapport à la norme dominante. Massad nous invite à nous méfier de certains stéréotypes orientalisants (le jeune Arabe est le jouet sexuel de l'homme blanc, la victime à libérer) ou de généralisations hâtives et anhistoriques sur le rapport entre homosexualité (occidentale) et islam (art. cit.). Chouchou, qui met en scène un couple franco-maghrébin, récuse précisément ce que ce genre de description pourrait impliquer. La masculinité dissidente de Chouchou n'est pas représentée comme spécifiquement maghrébine ni comme une assimilation à une norme occidentale perçue comme un progrès démocratique sexuel. La recherche d'un modèle bricolé n'échappe pas à l'autodérision. Chouchou est aussi comique dans l'expression de sa domesticité féminine que dans les moments où le personnage tombe dans l'excès répertorié de la culture camp. Il ne cherche pas à inverser ou critiquer une identité culturelle ethnique qui serait à la fois son héritage (son bien) mais aussi une norme (contraignante). Chouchou ne nous propose pas l'image d'une homosexualité maghrébine qui aurait ou non ce nom-là et ne se demande pas si le couple que le personnage forme avec Stanislas est interracial ou interreligieux. Le film fait du mariage une fin en soi. L'après-mariage est une absence structurante qui fait de cette union entre un travesti maghrébin et un grand bourgeois de France une fiction construite selon des conventions du conte de fées traditionnel: l'histoire s'arrête au moment de la cérémonie, avant même que le besoin de clôture ne soit pris en charge par une nouvelle formule rituelle: «Ils se marièrent et eurent beaucoup d'enfants. » Dans Chouchou, le mariage est le futur immédiat que le spectateur peut écrire seul, sans que l'image ne vienne corroborer l'évidence. Que la cérémonie elle-même soit réussie est implicite, non vérifiée, comme si cet élément relevait de l'évidence; la double masculinité est un détail sans importance. L'intertextualité ou plutôt la loi du genre du conte de fées se double d'un réseau performatif, le récit contemporain réaliste, si bien que le mariage des deux hommes est inséparable d'autres types de norme. Chouchou et Stanislas sont plaqués non seulement sur un modèle hétérosexuel que le travestissement de Chouchou rend visuellement plausible, mais ils deviennent aussi des personnages retirés des normes sociales contemporaines, ils deviennent le prince et la princesse des temps immémoriaux. Le problème de savoir s'ils auront ou non des héritiers ou s'ils pourront en adopter est aussi peu pertinent que la question de savoir si les autres contraintes identitaires seront ou non capables de remettre en cause le mariage.

Princesse de contes de fées, Chouchou n'est plus cet émigré maghrébin qui se fait passer pour un réfugié chilien au mépris de toute vraisemblance. Est passée sous silence la question du rapport entre son personnage et ses convictions religieuses. Le film ne pose pas la question de son rapport à l'islam ni de l'hypothétique réaction de la famille de Stanislas, dont 
les convictions religieuses ne sont pas explicitées. Ce mariage pourrait être spectaculairement chrétien s'il n'était pas d'abord un conte de fée. Toute dissidence par rapport à certains récits de la masculinité est subordonnée au respect de la loi du genre.

La façon dont une communauté construit les mariages fictifs et débat de la légalité de certains mariages réels et de l'illégitimité de certains autres est liée. Mais ce lien entre performance fictive et performance théâtralisée au sein de la loi est mystérieux, c'est-à-dire que les récits qui nous aident à construire une fiction ou une politique réussie, capable de rencontrer le public qu'elle crée par cohérence interne, ne sont pas capables d'expliquer comment politique et fiction s'articulent. Or, présupposer cette ignorance est une limite structurante au sein d'une réflexion sur l'évolution du genre par rapport à la norme. Poser que le lien entre imaginaire et acte civique ou politique est complexe et problématique permet à l'analyste culturel d'éviter deux risques: celui de couper les trois mariages du réseau de questions dans lequel le contexte contemporain les enserre (la problématique des mariages dits « gay » et/ou des modifications que le pacs va continuer à subir ${ }^{12}$ ), et celui de les inféoder aux seules modalités d'expression ou de pensée que ledit contexte autorise par habitude, réitération ou solidification des catégories (faut-il, peut-on être pour ou contre?) ${ }^{13}$.

Ainsi, les trois mariages sont tout à fait capables de s'adresser à des publics radicalement différents. On peut imaginer par exemple que les spectateurs sont belges, hollandais ou espagnols (c'est-à-dire qu'ils vivent dans des États qui reconnaissent la validité d'un mariage entre deux personnes de même sexe), qu'ils sont français, anglais ou américains (et plus ou moins concernés par les discours médiatiques, scientifiques, politiques ou associatifs qui organisent la question des mariages gay) ou qu'ils vivent dans des cultures qui s'étonnent de voir les discours occidentaux présupposer que les catégories de genre sont globales. Le moment historique où une société découvre que l'on peut remettre en cause une évidence depuis longtemps naturalisée (un mariage suppose la présence d'un homme et d'une femme) ressemble à ces moments où la recherche académique (philosophique, sociologique ou théorique au sens large) se penche sur les processus extrêmement complexes qui permettent à une norme de fonctionner de façon tautologique et invisible, c'est-à-dire d'imposer des normes soidisant éternelles et évidentes qui sont en fait créées par tout l'appareil dont

12. Voir D. Borrillo, É. Fassin et M. Iacub, dir., Au-delà du PACS : l'expertise familiale à l'épreuve de l'homosexualité, Paris, PUF, 1999.

13. Éric Fassin remarque que l'on peut très bien adopter une position politique différente si l'on est en France ou aux États-Unis dans la mesure où ce qui peut d'abord apparaître comme la même problématique (pour ou contre les mariages gay) fait en fait intervenir, selon le contexte, des facteurs qui modifient complètement les données du problème. Voir notamment «Same Sex, Different Politics: "Gay Marriage" Debates in France and the United States », Public Culture, n 13, 2, 2001, p. 215-232. 
ces normes disposent ${ }^{14}$. Il serait au mieux comiquement optimiste et au pire dangereusement totalitaire d'imaginer que l'on puisse recourir directement à l'accumulation d'un savoir scientifique pour discréditer la norme sociale que la philosophie ou la sociologie ont critiquée. Il est clair que le seul élément que les trois mariages considérés ici ont en commun est qu'ils appartiennent à un récit qui revendique le statut de fiction et qu'ils ne constituent pas des exemples de pratique sociale passée ou contemporaine. Pour autant, les propositions que ces trois exemples nous font sur la masculinité ne relèvent pas seulement d'une analyse des techniques de représentation et il n'est ni utile ni souhaitable de déclarer impertinents les outils de la sociologie, de la philosophie ou les voix qui mettent leurs travaux au service d'une cause politique. En d'autres termes, la façon dont ces récits parlent de la masculinité nous permet d'éviter l'opposition entre analyse formaliste (apolitique) et réflexion politique (qui oublierait le rôle de l'imaginaire). Ce qui se dit de la masculinité, de la façon dont on tient à la faire évoluer, mais aussi de ses frontières et de sa multiplicité intrinsèque passe toujours par une performance. Et en analysant les contraintes de cette performance, en d'autres termes en surveillant les différentes lois du genre que chaque performance reconnaît, il est possible de considérer en même temps les récits (dont la performance est liée à un acte) de fiction et (ceux dont la performance est régie par) un discours qui s'appelle le droit, la loi ou la politique.

\author{
Mireille Rosello \\ Université d'Amsterdam, Pays-Bas
}

\begin{abstract}
14. Voir ici les textes de J. Butler, notamment Undoing Gender, New York et Abingdon, Routledge, 2004, p. 52-56, sur le caractère double de la norme qui contraint et autorise, et The Psychic Life of Power, Stanford, Stanford University Press, 1997, p. 83-105, sur le processus de subjectivation que l'auteur relit par rapport à Foucault et à la psychanalyse et qui est capable à la fois de former le sujet et de l'assujettir.
\end{abstract}

